\title{
Long-Term Cilostazol Treatment and Predictive Factors on Outcomes of Endovascular Intervention in Patients with Diabetes Mellitus and Critical Limb Ischemia
}

Chiu-Yang Lee $\cdot$ Tao-Cheng $\mathrm{Wu} \cdot$ Shing-Jong Lin

Received: May 3, 2020 / Published online: June 20, 2020

(C) The Author(s) 2020
Methods: In this study, 172 consecutive patients with CLI (Fontaine levels III-IV) received cilostazol treatment after successful endovascular intervention according to the angiosome concept, and their primary patency rates and cardiovascular and amputation events during a 24-month follow-up period were assessed.

Result: The 24-month primary patency rate, mortality rate, and amputation rate were better in the patients under long-term cilostazol treatment $(P<0.001, P=0.029$, and $P=0.014)$. Weighted multivariate Cox analyses with a propensity scoring-based method showed that long-term cilostazol treatment [hazard ratio (HR) $0.2,95 \%$ confidence interval (CI) $0.11-0.36, P<0.001]$, direct revascularization (DR) (HR 0.46, 95\% CI $0.28-0.74, P=0.002$ ), and supervised exercise (HR $0.4,95 \%$ CI $0.24-0.66, P<0.001$ ) were independently associated with primary patency. Patients with lower-extremity amputation (LEA)

\section{T.-C. Wu}

Cardiovascular Research Center, National YangMing University, Taipei, Taiwan

S.-J. Lin

Taipei Heart Institute, Taipei Medical University, Taipei, Taiwan

\section{S.-J. Lin $(\bowtie)$}

Department of Medical Research, Taipei Veterans General Hospital, Taipei, Taiwan e-mail: shingjong88@gmail.com
T.-C. Wu ( $\square)$

Division of Cardiology, Department of Medicine, Taipei Veterans General Hospital, Taipei, Taiwan e-mail: dcwu@vghtpe.gov.tw 
had a higher risk of coronary artery disease (CAD) and mortality. Cellulitis and neuropathy were independently associated with LEA events (cellulitis: HR 2.89, 95\% CI 1.66-5.05, $P<0.001$; neuropathy: HR $2.2,95 \%$ CI $1.31-3.7, P=0.003$ ). Conclusion: Our results showed that patients with DM who received cilostazol treatment for more than 3 months had significantly better outcomes and decreased amputation and mortality rates after DR, and cellulitis and neuropathy were highly associated with the risk of limb loss. A large-scale randomized trial should be conducted in the future to confirm these results. Trial Registration: Taipei Veterans General Hospital (TVGH) IRB no. 2013-08-020B. Registered 30 August 2013.

Keywords: Coronary artery disease; Critical limb ischemia; Diabetes mellitus; Indirect and direct revascularization; Lower-extremity amputation

\section{Key Summary Points}

Why carry out this study?

Despite improvements in endovascular interventions and multidisciplinary approaches, maintaining clinical outcomes and prevention of limb loss have become increasingly challenging in patients with DM and critical limb ischemia (CLI).

This prospective study investigated the associations of cilostazol treatment with clinical outcomes and predictive factors in patients with diabetes mellitus (DM) and critical limb ischemia (CLI) after endovascular revascularization of the affected angiosome.

\section{What was learned from the study?}

Weighted multivariate Cox analyses with a propensity scoring-based method showed that long-term cilostazol treatment, direct revascularization (DR), and supervised exercise were independently associated with primary patency. The 24 -month primary patency rate, mortality rate, and amputation rate were better in patients under long-term cilostazol treatment.
Our study concluded that cilostazol treatment for more than 3 months and direct revascularization of the affected angiosome should be considered for patients with DM and CLI to maximize clinical outcomes and prevent further tissue loss. A large-scale randomized trial should be conducted in the future to confirm these results.

\section{INTRODUCTION}

Currently, approximately 2.3 million individuals in Taiwan have been diagnosed with diabetes mellitus (DM), the equivalent of 1 in 10 adults. The annual diagnosis rate of approximately 25,000 cases continues to increase according to figures reported by the Health Promotion Administration, Ministry of Health and Welfare. Type $2 \mathrm{DM}$ is associated with increased risks of macrovascular complications (such as stroke, cardiovascular disease, and heart failure) and microvascular complications (including retinopathy, neuropathy, lower-limb amputation, and kidney failure). Diabetic foot (DM foot) combined with ischemia, namely critical limb ischemia (CLI), is a severe form of peripheral arterial occlusive disease (PAOD) caused by atherosclerosis, which is similar to other cardiovascular diseases, and thereby may act as a predictor of premature cardiovascular events [1-5]. Patients with DM who have PAOD usually present with atypical leg pain or intermittent claudication, and do not receive a timely diagnosis and treatment until the disease progresses [2, 6, 7]. DM foot with CLI manifests as ischemic ulceration, gangrene, and rest pain, and is associated with high rates of cardiovascular mortality and lower-extremity amputation (LEA) [8, 9]; urgent revascularization is frequently required for limb salvage and preservation of quality of life. Despite recent advances in surgical and endovascular techniques, the clinical outcome of CLI in patients with DM is still inconsistent. 
Numerous studies have reported that individuals with DM foot and CLI have multiple predisposing factors and comorbidities, such as cellulitis, neuropathy, coronary artery disease (CAD), stroke, and chronic kidney disease (CKD), which can complicate treatment and potentially worsen the clinical outcome [10-15].

Even in patients who undergo successful revascularization for limb salvage, most still have claudication after the operation. Cilostazol treatment has been routinely applied in patients with PAOD and DM to improve intermittent claudication and increase their walking distance. Additionally, recent papers have suggested that cilostazol treatment may improve stent patency in coronary arteries or peripheral arteries [16-20]. To the best of our knowledge, information regarding the clinical outcomes of cilostazol treatment in the management of CLI is limited. Therefore, we hypothesized that long-term cilostazol treatment may improve the clinical outcome of patients with DM and CLI who receive endovascular intervention of the affected angiosome, along with risk factor modification, supervised exercise, prompt management of infections, and aggressive wound care.

Therefore, the purpose of this study was to investigate the associations of cilostazol treatment and predictive factors with maximization of beneficial clinical outcomes and maintenance of limb function in patients with DM and CLI after endovascular revascularization of the affected angiosome.

\section{METHODS}

\section{Study Population}

The Institutional Review Board (IRB) of Taipei Veterans General Hospital, Taiwan, approved this prospective, non-randomized study (IRB no. 2013-08-020B), in agreement with the Declaration of Helsinki. All participants provided written informed consent to participate in this study. From September 2013 to December 2017, 205 patients with DM and CLI were treated in a single medical center; of these, 33 patients did not satisfy the inclusion criteria, resulting in 172 patients being included in this study (Fig. 1). The inclusion criteria were patients aged 20-90 years; those with a first diagnosis of PAOD; those with DM; those with CLI (Fontaine class III and IV); those with tissue loss associated with an ankle pressure less than $70 \mathrm{mmHg}$ or a toe pressure less than $50 \mathrm{mmHg}$; those who underwent infrapopliteal artery intervention alone or combined with femoropopliteal or iliofemoral artery intervention; those who underwent successful endovascular revascularization (defined as less than 30\% residual stenosis on angiography, with evidence of a minimum of one tibial artery to the foot) [21]; those able and willing to perform regular exercise; and those who received cilostazol treatment after the intervention. Patients with acute artery embolism; those with Buerger's disease; those who had undergone prior amputation (minor or major); those with congestive heart failure (CHF) according to New York Heart Association (NYHA) function III-IV or an ejection fraction of less than 35\%; those with bleeding diathesis, acute cardiovascular diseases, or acute cerebrovascular diseases; and those with active diseases such as hepatitis, malignancy, or systemic infection were excluded.

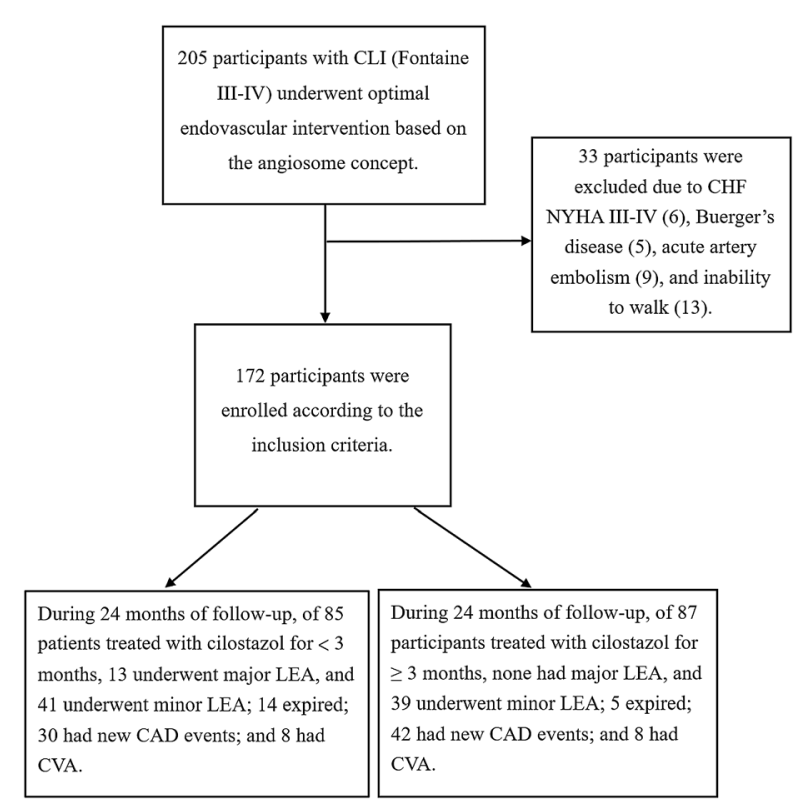

Fig. 1 Flow diagram of the study 
The National Health Insurance (NHI) administration suggests that cilostazol is indicated in patients with intermittent claudication, leg pain, or numbness related to PAOD in Taiwan.

Accordingly, after successful intervention, all patients were treated with cilostazol, the duration of treatment being based on resolution of the symptoms of claudication, leg pain, and numbness. If patients had resolution or reduction of symptoms along with a normal ankle brachial index (ABI) or sonographic findings, cilostazol treatment would be administered for 2-3 months. If symptoms persisted or abnormal findings were detected, cilostazol treatment would be administered for more than 3 months.

Thus, the patients in this study were divided into control and cilostazol groups according to the duration of cilostazol treatment. Patients in the cilostazol (long-term) group were treated with cilostazol for more than 3 months, while participants in the control (short-term) group were treated with cilostazol for 2-3 months (at most 3 months).

\section{Study Design}

The diagnosis and validation of CLI (defined as a Fontaine stage III or higher) were confirmed at our outpatient clinic according to clinical evaluation, duplex scanning, or the ABI and computed tomography angiography (CTA) [21, 22]. Peripheral neuropathy was diagnosed using tests such as a nerve conduction test and electromyography. Diabetic retinopathy is confined to the retina, which is damaged as a result of DM. This medical condition was diagnosed by an ophthalmologist by initially performing a dilated exam, and then a comprehensive eye examination.

The anatomical inclusion criteria, according to CTA performed prior to intervention, were based on an infrapopliteal artery intervention alone or combined with femoropopliteal or iliofemoral artery intervention.

The severity of ischemia and the involved lesion were stratified on the basis of the Wound, Ischemia, Foot Infection classification system of the Society for Vascular Surgery and the Trans-
Atlantic Inter-Society Consensus for the Management of Peripheral Arterial Disease (TASC II) classification [21-23]. In this study, patients with severe arterial calcification were classified on the basis of the Peripheral Academic Research Consortium Lesion and Vessel Characteristics and Definitions [24-26].

Revascularization can be divided into direct revascularization (DR) and indirect revascularization (IR) according to the angiosome concept. DR provides adequate blood flow directly to the area of ischemic tissue in patients with CLI, whereas IR supplies blood flow through collaterals to the ischemic limb [27-29].

\section{Medication and Physical Management}

Dual antiplatelet therapy was administered to patients undergoing stent implantation for at least 3 months, and single antiplatelet therapy was given later. Single antiplatelet therapy was prescribed to patients undergoing a simple angioplasty. All patients in this study received cilostazol treatment $(50-100 \mathrm{mg}$ bid) after the intervention.

According to the practice guidelines and published reports of the American College of Cardiology/American Heart Association (ACC/ AHA) [30-32], all patients were advised to perform exercise or walk. After revascularization, a rehabilitation physician was consulted, who collaborated to check each patient's condition and arrange a series of exercise therapy interventions. All of the enrolled patients and their families were routinely questioned to determine whether the patients were compliant regarding exercise every month at the outpatient office or by telephone.

\section{Study Endpoints}

The primary endpoints were the primary patency rates at $6,12,18$, and 24 months; secondary patency rates at 12 and 24 months; and amputation rate at 24 months. LEA in this study was defined as minor (below the ankle) or major (above the ankle) amputation. The secondary endpoints were overall survival (OS) or overall mortality associated with cardiovascular events 
or sepsis as a result of DM foot, overall amputation-free survival, cerebrovascular accident (CVA) at 24 months, and CAD at 12 and 24 months. Notably, amputation-free survival (AFS) in this study was defined as the avoidance of minor and major amputation.

All patients underwent clinical, ABI, and Doppler ultrasound examinations upon discharge and after $3,6,12$, and 24 months. If abnormal findings were obtained, CTA or invasive angiography was performed. Patency failure was defined as (1) a decrease in the ABI by 0.10 during follow-up or a return to the preprocedure level; (2) duplex ultrasonography findings of at most a 50\% stenosis diameter of the treated segments according to a peak systolic velocity index (intrastenotic peak systolic/ prestenotic ratio) of less than 2.4; or (3) CTA or invasive angiography findings indicating a stenosis diameter of at most $50 \%$ or an area of stenosis of at most $70 \%[25,33]$.

\section{Statistical Analysis}

Continuous data are presented as mean $\pm \mathrm{SD}$, and categorical data are presented as percentages. The Kolmogorov-Smirnov test was used to analyze the normality of numerical variable distributions. With regard to numerical variables, the Mann-Whitney $U$ test and independent samples $t$ test were used to determine intergroup differences. Frequencies and categorical variables were compared using the chisquare test. Kaplan-Meier curves were obtained for time-to-patency analysis. Patients being followed-up were censored at the time of death, major amputation, or patency failure for cumulative patency.

Predisposing factors of primary patency and amputation at 24 months were confirmed through univariate Cox regression. Furthermore, these significant factors and potential factors with $P$ values of less than 0.15 were analyzed using a weighted multivariate Cox regression model to evaluate the associations with primary patency and amputation according to the average treatment effect (ATE) or the average treatment effect on the treated (ATT). Inverse probability weighting propensity scores
(PS) were calculated on the basis of disease severity and location using logistic regression to estimate the ATE or the ATT.

A $P$ value of less than 0.05 was deemed significant. All analyses were performed using SPSS version 22.0 (SPSS Inc., Chicago, IL, USA).

\section{RESULTS}

In total, 205 patients with DM and CLI were treated with endovascular revascularization at a single medical center, and 33 patients were excluded on the basis of the study criteria. All 172 patients who were eligible for inclusion were analyzed, and underwent a comprehensive follow-up for 24 months. The mean follow-up period was $3.46 \pm 1.2$ years. A flow diagram of this study is shown in Fig. 1.

Data on the baseline demographic and clinical characteristics of the patients associated with cilostazol treatment for at most 3 months and more than 3 months are summarized in Table 1. After successful interventions based on the angiosome concept, the patients were divided into two groups according to the duration of cilostazol treatment. The cilostazol group included 87 participants treated with cilostazol for more than 3 months (long-term: cilostazol group), while the control group included 85 subjects treated with cilostazol for at most 3 months (short-term: control group).

The study sample comprised 59 women and 113 men, with a mean age of $73.1 \pm 9.6$ years (range 49-90 years). The mean ages of the patients in the cilostazol and control groups were $\quad 73.9 \pm 9.7$ and $72.2 \pm 9.5$ years $(P=0.234)$, respectively. Overall, $146(84.9 \%)$, 87 (50.9\%), and $64(37.2 \%)$ patients had hypertension, hyperlipidemia, and a positive smoking status, respectively. In addition, 62 patients (36\%) had CAD at baseline, 73 (42.4\%) had end-stage renal disease (ESRD) requiring regular dialysis, 62 (36\%) had diabetic retinopathy, $81(47.1 \%)$ had neuropathy, and 100 (58.1\%) had cellulitis.

In a subgroup analysis of the duration of cilostazol treatment, $36(41.4 \%), 46(52.9 \%), 36$ (41.4\%), 43 (49.4\%), 36 (65.5\%), 59 (67.8\%), 38 $(43.7 \%)$, and $35(40.2 \%)$ patients in the 
Table 1 Baseline demographic data and characteristics associated with cilostazol treatment for at most 3 months and more than 3 months

\begin{tabular}{|c|c|c|c|c|}
\hline Characteristic & $\begin{array}{l}\text { Total population } \\
(n=172)\end{array}$ & Control group $(n=85)$ & $\begin{array}{l}\text { Cilostazol group } \\
(n=87)\end{array}$ & $P$ value \\
\hline Age, years & $73.1 \pm 9.6$ & $72.2 \pm 9.5$ & $73.9 \pm 9.7$ & 0.234 \\
\hline BMI & $24.8 \pm 3.8$ & $25 \pm 3.4$ & $24.5 \pm 4.2$ & 0.431 \\
\hline SBP & $136.1 \pm 20.9$ & $136.9 \pm 17.7$ & $135.3 \pm 23.7$ & 0.632 \\
\hline Gender (female) & $60(34.9)$ & $34(40)$ & $26(29.9)$ & 0.164 \\
\hline Lesion site (left) & $98(57.0)$ & $49(57.6)$ & $49(56.3)$ & 0.861 \\
\hline Baseline CAD & $62(36.0)$ & $33(38.8)$ & $29(33.3)$ & 0.453 \\
\hline ESRD (regular dialysis) & $73(42.4)$ & $37(43.5)$ & $36(41.4)$ & 0.775 \\
\hline Hypertension & $146(84.9)$ & $74(87.1)$ & $72(82.8)$ & 0.431 \\
\hline Hyperlipidemia & $87(50.9)$ & $43(51.2)$ & $44(50.6)$ & 0.936 \\
\hline Baseline CVA & $25(14.5)$ & $14(16.5)$ & $11(12.6)$ & 0.476 \\
\hline Smoking & $64(37.2)$ & $33(38.8)$ & $31(35.6)$ & 0.665 \\
\hline Cellulitis on presentation & $100(58.1)$ & $54(63.5)$ & $46(52.9)$ & 0.157 \\
\hline Atrial fibrillation & $23(13.4)$ & $8(9.4)$ & $15(17.2)$ & 0.131 \\
\hline Baseline ABI & $0.52 \pm 0.15$ & $0.51 \pm 0.15$ & $0.53 \pm 0.15$ & 0.473 \\
\hline Calcification & $75(43.6)$ & $36(42.4)$ & $39(44.8)$ & 0.744 \\
\hline Retinopathy & $62(36)$ & $26(30.6)$ & $36(41.4)$ & 0.141 \\
\hline Neuropathy & $81(47.1)$ & $38(44.7)$ & $43(49.4)$ & 0.535 \\
\hline \multicolumn{5}{|l|}{ Fontaine classification } \\
\hline Stage III & $48(27.9)$ & $20(23.5)$ & $28(32.2)$ & 0.206 \\
\hline Stage IV & $124(72.1)$ & $65(76.5)$ & $59(67.8)$ & \\
\hline hsCRP (mg/L) & $4.03 \pm 5.96$ & $4.32 \pm 6.1$ & $3.75 \pm 5.84$ & 0.528 \\
\hline HbAlc (\%) & $7.65 \pm 1.56$ & $7.58 \pm 1.79$ & $7.71 \pm 1.34$ & 0.591 \\
\hline \multicolumn{5}{|c|}{ Concomitant medications } \\
\hline OHA alone & $65(37.8)$ & $27(31.8)$ & $38(43.7)$ & 0.107 \\
\hline Insulin alone & $78(45.3)$ & $43(50.6)$ & $35(40.2)$ & 0.172 \\
\hline Insulin duration, years & $4.24 \pm 3.9$ & $4.4 \pm 3.7$ & $4.1 \pm 4.1$ & 0.576 \\
\hline
\end{tabular}

$B M I$ body mass index, $S B P$ systolic blood pressure, $C A D$ coronary artery disease, $D M$ diabetes mellitus, ESRD end-stage renal disease, $C V A$ cerebrovascular accident, $\mathrm{ABI}$ ankle brachial index, hsCRP high-sensitivity C-reactive protein, HbAIc hemoglobin Alc, OHA oral hypoglycemic agent

cilostazol group had ESRD $(P=0.775)$, cellulitis $(P=0.157)$, retinopathy $(P=0.141)$, neuropathy $(P=0.535), \quad$ Fontaine stage IV CLI
$(P=0.206)$, sole use of an oral hypoglycemic agent (OHA) $(P=0.107)$, and insulin use alone $(P=0.172)$, respectively. The mean level of 
high-sensitivity C-reactive protein (hsCRP) was $4.32 \pm 6.1$ and $3.75 \pm 5.84$ in the control and cilostazol groups, respectively $(P=0.528)$. No significant difference was noted with regard to the hemoglobin A1c (HbA1c) value in the control and cilostazol groups $(P=0.591)$.

All diseased arteries were characterized using pretreatment imaging results on the basis of the stratification of lesions as per the updated 2015 TASC II classification for aortoiliac, femoropopliteal, and infrapopliteal lesions. As presented in Table 2, the patients in the cilostazol and control groups had multiple lesions. There were no significant differences with regards to aortoiliac, femoropopliteal, and infrapopliteal lesions between the two groups.

Data on the baseline demographic and clinical characteristics of the patients associated with amputation are summarized in Supplementary Table 1 . The mean ages of the patients in the no LEA (NLEA) and LEA groups were
$74.9 \pm 9.4$ and $71.5 \pm 9.5$ years, respectively $(P=0.018) ; 51.6 \%$ (48), 77.4\% (72), 44.1\% (41), $63.4 \%$ (59), 96.8\% (90), 21.5\% (20), and 59.1\% (55) of the patients in the LEA group had ESRD $(P=0.008)$, cellulitis $(P<0.001)$, retinopathy $(P=0.017)$, neuropathy $(P<0.001)$, Fontaine stage IV CLI $(P<0.001)$, OHA use alone $(P<0.001)$, and insulin use alone $(P<0.001)$, respectively. The mean level of hsCRP was $1.96 \pm 3.14$ and $5.79 \pm 7.13$ in the NLEA and LEA groups, respectively $(P<0.001)$. The mean level of HbA1c was $7.48 \pm 1.49$ and $7.78 \pm 1.61$ in the NLEA and LEA groups, respectively $(P=0.215)$.

\section{Endpoints}

The associations of the duration of cilostazol treatment with study endpoints at 24 months for all participants and subgroups are summarized in Table 3 . In this study, $58(33.7 \%)$ and

Table 2 Summary of lesion characteristics and distribution of diseased vessels in patients receiving cilostazol treatment for at most 3 months and more than 3 months

\begin{tabular}{llll}
\hline $\begin{array}{l}\text { Lesion characteristics } \\
\text { TASC II classification }\end{array}$ & Control group $(\boldsymbol{n}=\mathbf{8 5})$ & Cilostazol group $(\boldsymbol{n}=\mathbf{8 7})$ & P value \\
\hline Aortoiliac lesions & $13(15.3)$ & $12(13.8)$ & 0.337 \\
A & $34(40)$ & $26(29.9)$ & \\
B & $7(8.2)$ & $6(6.9)$ & \\
C & $0(0)$ & $2(2.3)$ & 0.333 \\
D & & & \\
Femoropopliteal lesions & $6(7.1)$ & $2(2.3)$ & \\
A & $15(17.6)$ & $22(25.3)$ & \\
B & $42(49.4)$ & $43(49.4)$ & \\
C & $22(25.9)$ & $20(23)$ & \\
D & & $22(25.3)$ & \\
Infrapopliteal lesions & $17(20)$ & $36(41.4)$ & \\
B & $40(47.1)$ & $29(33.3)$ & \\
C & $28(32.9)$ & & \\
D & Co. & & \\
\hline
\end{tabular}

TASC Trans-Atlantic Inter-Society Consensus for the Management of Peripheral Arterial Disease 
Table 3 Cilostazol Associations of cilostazol treatment for at most 3 months and more than 3 months with study endpoints at 24 months

\begin{tabular}{|c|c|c|c|c|}
\hline Parameters & $\begin{array}{l}\text { Total population } \\
(n=172)\end{array}$ & Control group $(n=85)$ & Cilostazol group $(n=87)$ & $P$ value \\
\hline Successful interventions & $172(100)$ & $85(100)$ & $87(100)$ & \\
\hline Simple angioplasty & $58(33.7)$ & $30(35.3)$ & $28(32.2)$ & \\
\hline Stent implantation & $114(66.3)$ & $55(64.7)$ & $59(67.8)$ & 0.666 \\
\hline Direct revascularization & $129(75)$ & $59(69.4)$ & $70(80.5)$ & 0.094 \\
\hline $\begin{array}{l}\text { Indirect } \\
\text { revascularization }\end{array}$ & $43(25)$ & $26(30.6)$ & $17(19.5)$ & \\
\hline \multicolumn{5}{|l|}{ Post-intervention ABI } \\
\hline 1 month & & $0.91 \pm 0.1$ & $0.95 \pm 0.16$ & 0.103 \\
\hline 6 months & & $0.82 \pm 0.18$ & $0.94 \pm 0.22$ & $<0.001$ \\
\hline \multicolumn{5}{|l|}{ Primary patency } \\
\hline 6 months & $148(88.6)$ & $67(82.7)$ & $81(94.2)$ & 0.02 \\
\hline 12 months & $120(71.9)$ & $44(54.3)$ & $76(88.4)$ & $<0.001$ \\
\hline 18 months & $90(53.9)$ & $21(25.9)$ & $69(80.2)$ & $<0.001$ \\
\hline 24 months & $83(49.7)$ & $15(18.5)$ & $68(79.1)$ & $<0.001$ \\
\hline \multicolumn{5}{|l|}{ Secondary patency } \\
\hline 12 months & $133(77.3)$ & $51(60)$ & $82(94.3)$ & $<0.001$ \\
\hline 24 months & $109(63.4)$ & $36(42.4)$ & $73(83.9)$ & $<0.001$ \\
\hline AFSR at 24 months & $79(45.9)$ & $31(36.5)$ & $48(55.2)$ & 0.014 \\
\hline LEA at 24 months & $93(54.1)$ & $54(63.5)$ & $39(44.8)$ & \\
\hline Major & $13(7.6)$ & $13(15.3)$ & 0 & \\
\hline Minor & $80(46.5)$ & $41(48.2)$ & $39(44.8)$ & \\
\hline 24-month mortality & $19(11)$ & $14(16.5)$ & $5(5.7)$ & 0.029 \\
\hline 24-month survival & $153(89)$ & $71(83.5)$ & $82(94.3)$ & 0.029 \\
\hline 12-month CAD events & $35(20.3)$ & $15(17.6)$ & $20(23.0)$ & 0.384 \\
\hline 24-month CAD events & $72(41.9)$ & $30(35.3)$ & $42(48.3)$ & 0.084 \\
\hline 12-month CVA events & $11(6.4)$ & $4(4.7)$ & $7(8.0)$ & 0.535 \\
\hline 24-month CVA events & $16(9.5)$ & $8(9.8)$ & $8(9.3)$ & 0.92 \\
\hline
\end{tabular}

$A B I$ ankle brachial index, $A F S R$ amputation-free survival rate, $L E A$ lower-extremity amputation, $C A D$ coronary artery disease, $C V A$ cerebrovascular accident

$114(66.3 \%)$ of the 172 participants underwent angioplasty alone and angioplasty plus stent implantation, respectively. The two methods yielded no significant differences according to cilostazol subgroup analysis $(P=0.666)$. The two groups had comparable post-intervention 
ABI scores at 1 month $(P=0.103)$, whereas the patients in the cilostazol group had a better post-intervention ABI at 6 months $(P<0.001)$.

The overall primary patency rates of the patients were $88.6 \%$ (148), 71.9\% (120), 53.9\% (90), and $59.7 \%$ (83) at 6, 12, 18, and 24 months, respectively. However, in the cilostazol subgroup analysis, significant differences were observed in the primary patency rate between the two groups at $6,12,18$, and 24 months $(P=0.02, P<0.001, P<0.001$, and $P<0.001$, respectively). The secondary patency was better in the cilostazol group at 12 and 24 months $(P<0.001$ and $P<0.001$, respectively). The Kaplan-Meier curves for the timeto-primary cumulative patency analysis showed that the patients who received cilostazol treatment for more than 3 months had a better survival rate than the patients who received treatment for at most 3 months (log rank test, $P<0.001$; Fig. 2). The overall amputation-free survival rate was $45.9 \%(79 / 172)$, and the percentage of amputations was significantly lower

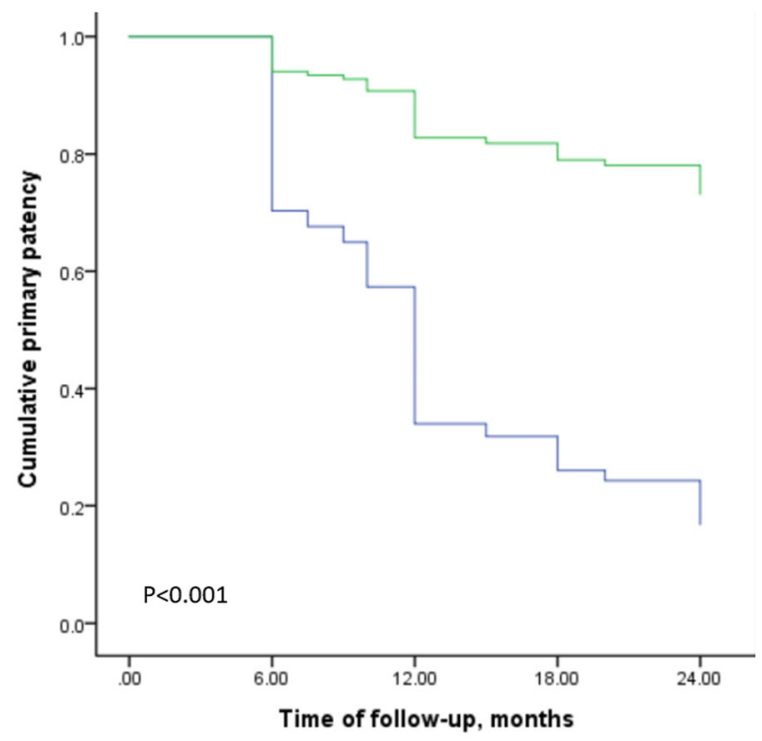

Number of patients at risk

$\begin{array}{llllll}-\leq 3 \mathrm{M} & 85 & 67 & 44 & 21 & 15 \\ ->3 \mathrm{M} & 87 & 81 & 76 & 69 & 68\end{array}$

Fig. 2 Patients who received cilostazol treatment for more than 3 months had a better patency rate than patients who received treatment for at most 3 months (log rank test, $P<0.001)$ in the cilostazol group than in the control group $(P=0.014)$. The 24 -month overall survival (OS) rate was $89 \%$ (153), and there was a significant difference between the control and cilostazol groups $(P=0.029)$. In addition, $20.3 \%$ $(n=35)$ and $41.9 \%(n=72)$ of the patients experienced CAD events at 12 and 24 months, respectively. No significant differences were detected in the occurrence of CAD at 12 and 24 months $(P=0.384$ and $P=0.084)$ between the control and cilostazol groups. Eleven and 16 CVA events were observed at 12 and 24 months, and no significant differences were observed with regards to CVA at 12 and 24 months between the two groups. Nineteen patients died during the 24-month follow-up period. Of these, nine patients died of cardiogenic shock related to ischemic heart disease and seven patients died owing to sepsis as a result of diabetes-related foot complications. The remaining three uremic patients died of complications of major surgery and sepsis related to pneumonia.

The treatment methods and study endpoints associated with amputation at 24 months for all participants and subgroups are summarized in Supplementary Table 2. Thus, $28(30.1 \%)$ and $65(69.9 \%)$ patients in the LEA group underwent angioplasty alone and angioplasty plus stent implantation, respectively. The two methods yielded no significant difference in LEA subgroup analysis $(P=0.277)$. A higher percentage of patients in the NLEA group had DR than in the LEA group $(P=0.017)$. In the LEA subgroup analysis, significant differences were observed in the primary patency rate between patients who did and did not undergo LEA at 12,18 , and 24 months $(P=0.011$, $P=0.001$, and $P=0.014$, respectively). The secondary patency was better in the patients without amputation at 12 months $(P<0.001)$. A total of $23.7 \%(n=22)$ and $49.5 \%(n=46)$ of the LEA group experienced CAD at 12 and 24 months, respectively. A significant difference in the occurrence of a CAD event at 24 months $(P=0.028)$ between the patients who did and did not undergo amputation was noted. No significant difference was observed in CVA between the two groups at 12 and 24 months. In total, 19 patients died during the 24-month follow-up period, 15 of whom had undergone 
LEA $(P=0.027)$. After the intervention and multidisciplinary management, the outcomes of tissue loss were followed up, including major amputations (13), minor amputations (80), and wound-healing (34).

\section{Assessment of Primary Patency}

In the univariate Cox regression analysis of the 24-month primary patency, long-term cilostazol usage (HR 0.18, 95\% CI 0.11-0.3, $P<0.001$ ), cellulitis (yes/no) (HR 1.86, 95\% CI 1.12-3.08, $P=0.016$ ), OHA use alone (HR 0.63, 95\% CI $0.39-1.0, P=0.051)$, duration of insulin use (HR $1.06,95 \%$ CI $1.0-1.13, P=0.045$ ), DR (HR 0.42 , 95\% CI 0.27-0.66, $P<0.001$ ), and exercise (HR $0.3,95 \%$ CI $0.19-0.46, P<0.001)$ were significantly associated with primary patency at 24 months (Table 4). Furthermore, the results of a weighted multivariate Cox regression model with the ATE showed that three main factors were independently associated with the 24-month primary patency: cilostazol treatment for more than 3 months (HR 0.2, 95\% CI $0.11-0.36, P<0.001$ ), DR (HR 0.46, 95\% CI $0.28-0.74, P=0.002$ ), and exercise (HR $0.4,95 \%$ CI 0.24-0.66, $P<0.001$; Table 4).

\section{Assessment of Amputation}

Univariate Cox regression analysis was first conducted to identify the clinical variables associated with amputation (Table 5). The following clinical factors were found to be associated with a relatively higher risk of LEA: cellulitis (HR 3.24, 95\% CI 1.99-5.28, $P<0.001$ ), DM retinopathy (HR $1.59,95 \% \mathrm{CI}$ $1.05-2.4, P=0.027$ ), neuropathy (HR 2.32, 95\% CI 1.51-3.55, $P<0.001)$, cilostazol treatment (> 3 months/ $\leq 3$ months) (HR 0.67, 95\% CI 0.45-1.02, $P=0.006$ ), insulin use alone (HR $2.08,95 \%$ CI $1.37-3.16, P<0.001)$, OHA use alone (HR 0.36, 95\% CI 0.22-0.59, $P<0.001$ ), duration of insulin treatment (HR 1.12, 95\% CI 1.06-1.18, $P<0.001)$, and no supervised exercise (HR 2.21, 95\% CI 1.46-3.34, $P<0.001$ ). Moreover, the significant and potential factors were analyzed using a weighted Cox regression model with the ATE method. Finally, cellulitis and neuropathy were found to be independently associated with amputation (cellulitis: HR 2.89, 95\% CI 1.66-5.05, $P<0.001$; neuropathy: HR $2.2,95 \%$ CI $1.31-3.7, P=0.003)$.

\section{DISCUSSION}

Treatment of diabetic CLI is more challenging as compared with treatment of proximal PAOD with claudication as a result of multiple risk factors in patients and diffuse tough calcification in infrapopliteal lesions. Despite great advances in surgical and endovascular techniques, the clinical outcome of CLI in patients with DM is still inconsistent. This study demonstrated that cilostazol treatment for more than 3 months combined with a multidisciplinary approach may improve the clinical outcome and prevent further tissue loss in patients with DM and CLI after endovascular revascularization of the affected angiosome.

Cilostazol is a selective inhibitor of phosphodiesterase type 3 (PDE III inhibitor). It has a therapeutic focus on the inhibition of phosphodiesterase activity and suppression of cAMP degradation, resulting in increased cAMP in platelets and blood vessels and leading to inhibition of platelet aggregation and vasodilation [34]. Cilostazol is indicated for the treatment of intermittent claudication in patients with PAOD $[35,36]$. In recent years, cilostazol has been used for the secondary prevention of brain infarction through its antiplatelet antithrombotic effects [37]. Furthermore, some studies have shown that cilostazol can prevent the proliferation of smooth muscle cells, consequently increasing peripheral blood flow and insulin sensitivity by reducing inflammation $[35,38]$. However, insufficient data are available to explain whether cilostazol increases the functional status of the involved lower extremities, improves primary patency in patients with CLI, as well as subsequent improvement in quality of life, and reduces the occurrence of adverse cardiovascular events and all-cause mortality. This study comprehensively demonstrated that cilostazol treatment for more than 3 months improved 
Table 4 Cox regression analysis of primary patency

\begin{tabular}{|c|c|c|c|c|}
\hline \multirow[t]{2}{*}{ Variable } & \multicolumn{2}{|l|}{ Univariate } & \multicolumn{2}{|l|}{ Multivariate $^{\mathbf{a}}$} \\
\hline & HR (95\% CI) & $P$ value & $\begin{array}{l}\text { ATE } \\
\text { HR (95\% CI) }\end{array}$ & $\begin{array}{l}\text { ATT } \\
\text { HR (95\% CI) }\end{array}$ \\
\hline Age, years & $1.0(0.98-1.03)$ & 0.697 & & \\
\hline Gender (F/M) & $1.51(0.99-2.32)$ & 0.059 & $0.93(0.57-1.54)$ & $0.98(0.59-1.65)$ \\
\hline BMI & $0.99(0.94-1.05)$ & 0.775 & & \\
\hline Smoking (yes/no) & $1.12(0.72-1.73)$ & 0.615 & & \\
\hline Hyperlipidemia (yes/no) & $1.45(0.94-2.23)$ & 0.095 & $1.35(0.85-2.16)$ & $1.43(0.89-2.3)$ \\
\hline CAD (yes/no) & $0.94(0.6-1.46)$ & 0.766 & & \\
\hline Hypertension (yes/no) & $0.8(0.52-1.67)$ & 0.803 & & \\
\hline ESRD (yes/no) & $0.98(0.64-152)$ & 0.942 & & \\
\hline Calcification (yes/no) & $1.4(0.92-2.15)$ & 0.118 & $1.25(0.75-2.07)$ & $1.14(0.67-1.92)$ \\
\hline Fontaine stage (IV/III) & $1.28(0.78-2.09)$ & 0.329 & & \\
\hline Cellulitis (yes/no) & $1.86(1.12-3.08)$ & 0.016 & $1.18(0.72-1.94)$ & $1.18(0.71-1.96)$ \\
\hline Neuropathy & $1.11(0.72-1.69)$ & 0.64 & & \\
\hline Ischemia severity & $1.26(0.78-2.02)$ & 0.341 & & \\
\hline \multicolumn{5}{|l|}{$\mathrm{ABI}<0.6 / \mathrm{ABI} \geq 0.6$} \\
\hline Statin (yes/no) & $1.25(0.81-1.93)$ & 0.304 & & \\
\hline Antiplatelet (yes/no) & $0.99(0.53-1.87)$ & 0.991 & & \\
\hline Cilostazol ( $>3$ months $/ \leq 3$ months $)$ & $0.18(0.11-0.3)$ & $<0.001$ & $0.2(0.11-0.36)$ & $0.21(0.11-0.38)$ \\
\hline Insulin alone (yes/no) & $1.42(0.93-2.17)$ & 0.11 & $0.80(0.39-1.66)$ & $0.79(0.37-1.69)$ \\
\hline OHA alone (yes/no) & $0.63(0.39-1.0)$ & 0.051 & $0.98(0.31-3.09)$ & $0.88(0.26-2.94)$ \\
\hline Duration of insulin, years & $1.06(1.0-1.13)$ & 0.045 & $0.98(0.89-1.09)$ & $0.98(0.88-1.08)$ \\
\hline Involved leg (L/R) & $0.8(0.53-1.23)$ & 0.313 & & \\
\hline Intervention (PTA/stenting) & $0.83(0.52-1.33)$ & 0.44 & & \\
\hline $\mathrm{DR} / \mathrm{IR}$ & $0.42(0.27-0.66)$ & $<0.001$ & $0.46(0.28-0.74)$ & $0.41(0.28-0.75)$ \\
\hline Exercise (yes/no) & $0.3(0.19-0.46)$ & $<0.001$ & $0.4(0.24-0.66)$ & $0.41(0.24-0.68)$ \\
\hline
\end{tabular}

$H R$ hazard ratio, $C I$ confidence interval, BMI body mass index, CAD coronary artery disease, DM diabetes mellitus, ESRD end-stage renal disease, $D R$ direct revascularization, IR indirect revascularization, $P T A$ percutaneous transluminal angioplasty, $A B I$ ankle brachial index, $O H A$ oral hypoglycemic agent

a Multivariate weighted Cox regression with average treatment effect (ATE) or average treatment effect on the treated (ATT)

the 6-, 12-, 18-, and 24-month primary patency, subsequently promoting wound-healing and decreasing the risk of LEA as compared with cilostazol treatment for at most 3 months. Notably, recent studies reporting that cilostazol treatment may improve stent patency in 
Table 5 Cox regression analysis of risk factors associated with amputation

\begin{tabular}{|c|c|c|c|c|}
\hline \multirow[t]{2}{*}{ Variable } & \multicolumn{2}{|l|}{ Univariate } & \multicolumn{2}{|l|}{ Multivariate $^{a}$} \\
\hline & HR (95\% CI) & $P$ value & $\overline{\text { ATE }}$ & ATT \\
\hline Age, years & $0.98(0.96-1.0)$ & 0.076 & $0.99(0.97-1.01)$ & $0.99(0.97-1.02)$ \\
\hline Gender $(\mathrm{F} / \mathrm{M})$ & $1.17(0.77-1.78)$ & 0.457 & & \\
\hline BMI & $0.99(0.94-1.04)$ & 0.681 & & \\
\hline Smoking (yes/no) & $1.04(0.68-1.59)$ & 0.852 & & \\
\hline Hyperlipidemia (yes/no) & $1.0(0.66-1.5)$ & 0.988 & & \\
\hline Baseline CAD (yes/no) & $0.69(0.45-1.07)$ & 0.097 & $0.8(0.52-1.23)$ & $0.78(0.5-1.22)$ \\
\hline Hypertension (yes/no) & $0.67(0.39-1.16)$ & 0.155 & & \\
\hline ESRD (yes/no) & $1.41(0.94-2.11)$ & 0.1 & $0.83(0.53-1.3)$ & $0.82(0.52-1.3)$ \\
\hline Cellulitis (yes/no) & $3.24(1.99-5.28)$ & $<0.001$ & $2.89(1.66-5.05)$ & $2.98(1.69-5.25)$ \\
\hline Retinopathy (yes/no) & $1.59(1.05-2.4)$ & 0.027 & $1.36(0.8-2.32)$ & $1.44(0.84-2.48)$ \\
\hline Neuropathy (yes/no) & $2.32(1.51-3.55)$ & $<0.001$ & $2.2(1.31-3.7)$ & $2.29(1.36-3.84)$ \\
\hline Calcification (yes/no) & $1.24(0.81-1.91)$ & 0.324 & & \\
\hline Ischemia severity & $1.11(0.72-1.7)$ & 0.643 & & \\
\hline \multicolumn{5}{|l|}{$\mathrm{ABI}<0.6 / \mathrm{ABI} \geq 0.6$} \\
\hline Interventions & $0.84(0.54-1.31)$ & 0.434 & & \\
\hline \multicolumn{5}{|l|}{ (PTA/stent) } \\
\hline IR/DR & $1.51(0.98-2.34)$ & 0.063 & $1.25(0.82-1.9)$ & $1.14(0.75-1.74)$ \\
\hline Statin (yes/no) & $0.93(0.62-1.39)$ & 0.71 & & \\
\hline Antiplatelet (yes/no) & $0.83(0.45-1.52)$ & 0.548 & & \\
\hline Cilostazol ( $>3$ months $/ \leq 3$ months) & $0.67(0.45-1.02)$ & 0.006 & $0.78(0.49-1.24)$ & $0.77(0.49-1.23)$ \\
\hline Insulin alone (yes/no) & $2.08(1.37-3.16)$ & $<0.001$ & $0.81(0.46-1.4)$ & $0.73(0.42-1.26)$ \\
\hline OHA alone (yes/no) & $0.36(0.22-0.59)$ & $<0.001$ & $0.59(0.22-1.59)$ & $0.56(0.21-1.51)$ \\
\hline Insulin duration, years & $1.12(1.06-1.18)$ & $<0.001$ & $1.05(0.95-1.17)$ & $1.05(0.95-1.16)$ \\
\hline Exercise (no/yes) & $2.21(1.46-3.34)$ & $<0.001$ & $0.99(0.56-1.74)$ & $1.01(0.57-1.78)$ \\
\hline Involved leg $(\mathrm{L} / \mathrm{R})$ & $0.91(0.61-1.37)$ & 0.651 & & \\
\hline
\end{tabular}

$H R$ hazard ratio, $C I$ confidence interval. $B M I$ body mass index, $C A D$ coronary artery disease, $D M$ diabetes mellitus, ESRD end-stage renal disease, $D R$ direct revascularization, $I R$ indirect revascularization, $P T A$ percutaneous transluminal angioplasty, $A B I$ ankle brachial index, $O H A$ oral hypoglycemic agent

${ }^{2}$ Multivariate weighted Cox regression with average treatment effect (ATE) or average treatment effect on the treated (ATT)

coronary or peripheral arteries $[16,17,19]$ were in accordance with our preliminary findings; that is, cilostazol treatment can improve the primary patency and further decrease the LEA and mortality rates in patients with CLI and DM. 
Another concern was the role of supervised exercise in patients with CLI and DM. Numerous studies have indicated that exercise rehabilitation improves the functional outcomes and peripheral circulation of patients with PAOD presenting with intermittent claudication [39, 40]. The 2016 ACC/AHA guidelines state that exercise plays a key role in the treatment of claudication, with substantial improvements reported in functional status [41]. This guideline supported our results that a supervised exercise program was an independent factor influencing the 24-month primary patency, which consequently may have decreased the LEA risk in patients with DM and CLI (Fig. 2, Table 3, and Supplementary Tables 1 and 2).

Another concern was revascularization based on the angiosome concept. Some studies have shown that the improvements of wound-healing and the major amputation rate after IR in the presence of collaterals were similar to the outcomes after DR in patients with CLI $[16,17,19,42]$. Accordingly, our results indicated that DR based on the affected angiosome is an important factor affecting the primary patency and decreasing the risk of LEA.

People with DM and CLI are susceptible to foot problems, manifested as several characteristic pathologies such as infection, neuropathy, diabetic foot ulcer, ischemic pain, and gangrene change, which cause poor blood circulation to the lower limb, finally leading to amputation. Some studies have reported that CLI combined with diabetic foot ulcer is a major cause of morbidity, accounting for approximately twothirds of all non-traumatic amputations performed in the USA [43, 44]. The causes of amputations are multifactorial, and diabetic neuropathy and cellulitis were independent predisposing factors associated with the risk of LEA according to the results of weighted multivariate Cox analysis. Diabetic neuropathy is nerve damage or dysfunction, frequently resulting from poor control of a high sugar level and subsequent impairment of microcirculation. Nerve damage usually decreases the peripheral sensation in the lower limbs, which can cause unnoticed injuries of the foot, manifested with skin ulcers or infections. In this study, of the 81 patients with diabetic neuropathy, $72.8 \%$ (59) had LEAs $(P<0.001$, Supplementary Table 1). Neuropathy, intractable infection, and severe PAOD may compromise microcirculation and macrocirculation of the lower limbs, breaking down the tissue and finally leading to amputation.

The results obtained after a 24-month follow-up period demonstrated that 93 (54.1\%) patients required LEA, with $80(46.5 \%)$ requiring minor amputation and 13 (7.6\%) requiring major amputation. The rate of major LEA in this study was considerably lower than the corresponding rate observed in some previous trials [43-45]. Furthermore, during the 24-month follow-up period, the risk of LEA was highly associated with newly developed ischemic heart disease, with $49.5 \%(n=46)$ of the patients who underwent LEA developing this disease as compared with $32.9 \%(n=26)$ of the patients who did not undergo LEA $(P=0.028)$. The mortality rate of the patients who underwent LEA was $16.1 \%(n=15)$, as compared with a mortality rate of $5.1 \%(n=4)$ in the patients who did not undergo LEA $(P=0.027)$. The patients with DM who underwent LEA had a 1.5-fold and 3-fold higher risk of new CAD events and mortality events, respectively, as compared with the patients who did not undergo LEA. These results indicated that amputation is a lethal medical condition, and that patients undergoing LEA experience markedly more cardiovascular events and have a higher mortality rate than patients who do not undergo LEA. Therefore, prevention of diabetic foot ulcer is a key measure; aggressive treatment of diabetic foot ulcers is challenging and prolonged and requires a multidisciplinary team and multiple approaches to preserve limb function, decrease cardiovascular complications, and promote quality of life $[45,46]$.

Patients with insulin resistance or poor control of their sugar level are more likely to develop microvascular complications (including retinopathy, neuropathy, kidney failure, and diabetic foot), and later macrovascular complications (such as stroke, cardiovascular disease, and heart failure). In this study, we found that over half of LEAs were attributable to a combination of DM, neuropathy, 
calcification, cellulitis and ESRD. DM is a chronic illness that causes endothelial and platelet dysfunction. In particular, DM combined with CKD is a leading cause of vascular calcification (VC), particularly medial arterial calcification (Mönckeberg's medial sclerosis). Numerous studies have proposed that VC is most common in patients with DM and CKD, and that it is associated with increased cardiovascular morbidity and mortality $[45,47-50]$.

According to subgroup analysis, 73 (42.4\%) and $75(43.6 \%)$ patients with DM had ESRD and calcification of the involved arteries, respectively, and 31 of 43 patients with ESRD and calcification had undergone LEA $(P=0.006$, Supplementary Table 1). These novel findings indicated that arterial calcification is associated with the risk of LEA in patients with DM undergoing dialysis. Owing to the small size of the calcification subgroup, further studies are warranted to verify the associations of arterial calcification with cardiovascular and LEA risks.

This study had some potential limitations that should be addressed. The first limitation was that it was a non-randomized study that did not provide sufficient power for extensive statistical comparisons. To decrease the bias, a PSweighted multivariate Cox regression model was used to evaluate the associations with primary patency and amputation based on disease severity and location. The second limitation was the small number of patients with DM and CLI owing to the strict inclusion criteria that all patients must have received cilostazol treatment after successful revascularization. The third limitation was that the reports of subgroup analyses were not head-to-head or matched comparisons. More longitudinal, comparative clinical trials should be conducted with larger sample sizes to further investigate the relative outcomes and mortality in patients with DM and CLI.

\section{CONCLUSION}

Patients with DM who received cilostazol treatment for more than 3 months had significantly better outcomes and decreased amputation rates after direct revascularization of the affected angiosome. Furthermore, cellulitis and diabetic neuropathy were the main predisposing factors for the occurrence of LEA. Future longitudinal and comparative clinical trials should be conducted with larger sample sizes to further investigate the associations of cilostazol treatment with the relative outcomes and mortality in patients with DM and CLI.

\section{ACKNOWLEDGEMENTS}

We thank the participants of this study.

Funding. No funding or sponsorship was received for this study or publication of this article. The Rapid Service Fee was funded by the authors.

Authorship. All named authors meet the International Committee of Medical Journal Editors (ICMJE) criteria for authorship for this article, take responsibility for the integrity of the work as a whole, and have given their approval for this version to be published.

Authorship Contributions. CYL, TCW, and SJL contributed to conceptualization and investigation. CYL planned the data curation and analysis. SJL and TCW performed the validation. CYL prepared the original draft. All authors reviewed and revised the manuscript. All authors approved the final version of the manuscript.

Disclosures. Chiu-Yang Lee, Tao-Cheng Wu, and Shing-Jong Lin have nothing to disclose.

Compliance with Ethics Guidelines. The Institutional Review Board (IRB) of Taipei Veterans General Hospital, Taiwan, approved this prospective, non-randomized study (IRB no. 2013-08-020B), in agreement with the Declaration of Helsinki. All participants provided written informed consent to participate in this study.

Data Availability. The datasets during the current study are available from the corresponding author on reasonable request. 
Open Access. This article is licensed under a Creative Commons Attribution-NonCommercial 4.0 International License, which permits any non-commercial use, sharing, adaptation, distribution and reproduction in any medium or format, as long as you give appropriate credit to the original author(s) and the source, provide a link to the Creative Commons licence, and indicate if changes were made. The images or other third party material in this article are included in the article's Creative Commons licence, unless indicated otherwise in a credit line to the material. If material is not included in the article's Creative Commons licence and your intended use is not permitted by statutory regulation or exceeds the permitted use, you will need to obtain permission directly from the copyright holder. To view a copy of this licence, visit http:// creativecommons.org/licenses/by-nc/4.0/.

\section{REFERENCES}

1. Sutton-Tyrrell K, Venkitachalam L, Kanaya AM, et al. Relationship of ankle blood pressures to cardiovascular events in older adults. Stroke. 2008;39: 863-9.

2. Hirsch AT, Criqui MH, Treat-Jacobson D, et al. Peripheral arterial disease detection, awareness, and treatment in primary care. JAMA. 2001;286: 1317-24.

3. McDermott MM, Kerwin DR, Liu K, et al. Prevalence and significance of unrecognized lower extremity peripheral arterial disease in general medicine practice. J Gen Intern Med. 2001;16:384-90.

4. Murabito JM, Evans JC, Nieto K, Larson MG, Levy D, Wilson PW. Prevalence and clinical correlates of peripheral arterial disease in the Framingham Offspring Study. Am Heart J. 2002;143:961-5.

5. McDermott MM, Greenland P, Liu K, et al. Leg symptoms in peripheral arterial disease: associated clinical characteristics and functional impairment. JAMA. 2001;286:1599-606.

6. McDermott MM, Hoff F, Ferrucci L, et al. Lower extremity ischemia, calf skeletal muscle characteristics, and functional impairment in peripheral arterial disease. J Am Geriatr Soc. 2007;55:400-6.
7. Jude EB, Eleftheriadou I, Tentolouris N. Peripheral arterial disease in diabetes-a review. Diabet Med. 2010;27:4-14.

8. Rodrigues BT, Vangaveti VN, Malabu UH. Prevalence and risk factors for diabetic lower limb amputation: a clinic-based case control study. J Diabetes Res. 2016;2016:5941957.

9. Spreen MI, Gremmels H, Teraa M, et al. Diabetes is associated with decreased limb survival in patients with critical limb ischemia: pooled data from two randomized controlled trials. Diabetes Care. 2016;39:2058-2064.

10. Ho CY, Shanahan CM. Medial arterial calcification: an overlooked player in peripheral arterial disease. Arterioscler Thromb Vasc Biol. 2016;36:1475-82.

11. Lau WL, Ix JH. Clinical detection, risk factors, and cardiovascular consequences of medial arterial calcification: a pattern of vascular injury associated with aberrant mineral metabolism. Semin Nephrol. 2013;33:93-105.

12. Norgren L, Hiatt WR, Dormandy JA, et al. Intersociety consensus for the management of peripheral arterial disease (TASC II). J Vasc Surg. 2007;45 Suppl S:S5-67.

13. Chen J, Mohler ER 3rd, Garimella PS, et al. Ankle brachial index and subsequent cardiovascular disease risk in patients with chronic kidney disease. J Am Heart Assoc. 2016;5:e003339.

14. Chen HY, Wei F, Wang LH, et al. Abnormal anklebrachial index and risk of cardiovascular or allcause mortality in patients with chronic kidney disease: a meta-analysis. J Nephrol. 2017;30: 493-501.

15. Feiring AJ. Footnotes on critical limb ischemia. J Am Coll Cardiol. 2008;51:1975-6.

16. Bangalore S, Singh A, Toklu B, et al. Efficacy of cilostazol on platelet reactivity and cardiovascular outcomes in patients undergoing percutaneous coronary intervention: insights from a meta-analysis of randomised trials. Open Heart. 2014;1: e000068.

17. Benjo AM, Garcia DC, Jenkins JS, et al. Cilostazol increases patency and reduces adverse outcomes in percutaneous femoropopliteal revascularisation: a meta-analysis of randomised controlled trials. Open Heart. 2014;1:e000154.

18. Chapman TM, Goa KL. Cilostazol: a review of its use in intermittent claudication. Am J Cardiovasc Drugs. 2003;3:117-38. 
19. Ishii H, Kumada $\mathrm{Y}$, Toriyama $\mathrm{T}$, et al. Cilostazol improves long-term patency after percutaneous transluminal angioplasty in hemodialysis patients with peripheral artery disease. Clin J Am Soc Nephrol. 2008;3:1034-40.

20. Zuliani Mauro MF, Mangione JA, Costa JR Jr, et al. Randomized angiographic and intravascular ultrasound comparison of dual-antiplatelet therapy vs triple-antiplatelet therapy to reduce neointimal tissue proliferation in diabetic patients. J Invasive Cardiol. 2017;29:76-81.

21. Cull DL, Manos G, Hartley MC, et al. An early validation of the Society for Vascular Surgery lower extremity threatened limb classification system. J Vasc Surg. 2014;60:1535-41.

22. Hardman RL, Jazaeri O, Yi J, Smith M, Gupta R. Overview of classification systems in peripheral artery disease. Semin Intervent Radiol. 2014;31: 378-88.

23. TASC Steering Committee, Jaff MR, White CJ, et al. An update on methods for revascularization and expansion of the TASC lesion classification to include below-the-knee arteries: a supplement to the inter-society consensus for the management of peripheral arterial disease (TASC II). Vasc Med. 2015;20:465-478.

24. Dattilo R, Himmelstein SI, Cuff RF. The COMPLIANCE 360 degrees Trial: a randomized, prospective, multicenter, pilot study comparing acute and long-term results of orbital atherectomy to balloon angioplasty for calcified femoropopliteal disease. J Invasive Cardiol. 2014;26:355-60.

25. Patel MR, Conte MS, Cutlip DE, et al. Evaluation and treatment of patients with lower extremity peripheral artery disease: consensus definitions from Peripheral Academic Research Consortium (PARC). J Am Coll Cardiol. 2015;65:931-41.

26. Rocha-Singh KJ, Zeller T, Jaff MR. Peripheral arterial calcification: prevalence, mechanism, detection, and clinical implications. Catheter Cardiovasc Interv. 2014;83:E212-20.

27. Iida $\mathrm{O}$, Soga $\mathrm{Y}$, Hirano $\mathrm{K}$, et al. Long-term results of direct and indirect endovascular revascularization based on the angiosome concept in patients with critical limb ischemia presenting with isolated below-the-knee lesions. J Vasc Surg. 2012;55(363-370):e365.

28. Jongsma H, Bekken JA, Akkersdijk GP, Hoeks SE, Verhagen HJ, Fioole B. Angiosome-directed revascularization in patients with critical limb ischemia. J Vasc Surg. 2017;65(1208-1219):e1201.
29. Lejay A, Georg Y, Tartaglia E, et al. Long-term outcomes of direct and indirect below-the-knee open revascularization based on the angiosome concept in diabetic patients with critical limb ischemia. Ann Vasc Surg. 2014;28:983-9.

30. Francia P, Gulisano M, Anichini R, Seghieri G. Diabetic foot and exercise therapy: step by step the role of rigid posture and biomechanics treatment. Curr Diabetes Rev. 2014;10:86-99.

31. Hamburg NM, Balady GJ. Exercise rehabilitation in peripheral artery disease: functional impact and mechanisms of benefits. Circulation. 2011;123: 87-97.

32. Hirsch AT, Haskal ZJ, Hertzer NR, et al. ACC/AHA 2005 Practice Guidelines for the management of patients with peripheral arterial disease (lower extremity, renal, mesenteric, and abdominal aortic). Circulation. 2006;113:e463-654.

33. Diehm N, Pattynama PM, Jaff MR, et al. Clinical endpoints in peripheral endovascular revascularization trials: a case for standardized definitions. Eur J Vasc Endovasc Surg. 2008;36:409-19.

34. Kawanabe $\mathrm{Y}$, Takahashi $\mathrm{M}$, Jin X, et al. Cilostazol prevents endothelin-induced smooth muscle constriction and proliferation. PLoS One. 2012;7: e44476.

35. Collinson DJ, Donnelly R. Cilostazol: improving walking distance in patients with intermittent claudication. Expert Rev Cardiovasc Ther. 2004;2: 503-9.

36. Hiatt WR. Medical treatment of peripheral arterial disease and claudication. N Engl J Med. 2001;344: 1608-21.

37. Tanahashi N. Antiplatelet therapy for secondary prevention of cerebral infarction [Article in Japanese]. Nihon Rinsho. 2014;72:1270-5.

38. Katakami N, Kim YS, Kawamori R, Yamasaki Y. The phosphodiesterase inhibitor cilostazol induces regression of carotid atherosclerosis in subjects with type 2 diabetes mellitus: principal results of the Diabetic Atherosclerosis Prevention by Cilostazol (DAPC) study: a randomized trial. Circulation. 2010;121:2584-91.

39. Gardner AW, Katzel LI, Sorkin JD, et al. Exercise rehabilitation improves functional outcomes and peripheral circulation in patients with intermittent claudication: a randomized controlled trial. J Am Geriatr Soc. 2001;49:755-62.

40. Mazari FA, Carradice D, Rahman MN, et al. An analysis of relationship between quality of life indices and clinical improvement following 
intervention in patients with intermittent claudication due to femoropopliteal disease. J Vasc Surg. 2010;52:77-84.

41. Gerhard-Herman MD, Gornik HL, Barrett C, et al. 2016 AHA/ACC guideline on the management of patients with lower extremity peripheral artery disease: a report of the American College of Cardiology/American Heart Association Task Force on Clinical Practice Guidelines. Circulation. 2017;135: e726-79.

42. Harwood AE, Cayton T, Sarvanandan R, Lane R, Chetter I. A review of the potential local mechanisms by which exercise improves functional outcomes in intermittent claudication. Ann Vasc Surg. 2016;30:312-20.

43. Abedin M, Tintut Y, Demer LL. Vascular calcification: mechanisms and clinical ramifications. Arterioscler Thromb Vasc Biol. 2004;24:1161-70.

44. Arambewela MH, Somasundaram NP, Jayasekara $\mathrm{H}$, et al. Prevalence of chronic complications, their risk factors, and the cardiovascular risk factors among patients with type 2 diabetes attending the diabetic clinic at a Tertiary Care Hospital in Sri Lanka. J Diabetes Res. 2018;2018:4504287.
45. Hippisley-Cox J, Coupland C. Diabetes treatments and risk of amputation, blindness, severe kidney failure, hyperglycaemia, and hypoglycaemia: open cohort study in primary care. BMJ. 2016;352:i1450.

46. Lai YJ, Hu HY, Lin CH, Lee ST, Kuo SC, Chou P. Incidence and risk factors of lower extremity amputations in people with type 2 diabetes in Taiwan, 2001-2010. J Diabetes. 2015;7:260-7.

47. Edmonds ME, Morrison N, Laws JW, Watkins PJ. Medial arterial calcification and diabetic neuropathy. Br Med J (Clin Res Ed). 1982;284:928-30.

48. Marso SP, Hiatt WR. Peripheral arterial disease in patients with diabetes. J Am Coll Cardiol. 2006;47: 921-9.

49. Nitta K. Vascular calcification in patients with chronic kidney disease. Ther Apher Dial. 2011;15: 513-21.

50. Pyörälä K, Laakso M, Uusitupa M. Diabetes and atherosclerosis: an epidemiologic view. Diabetes Metab Rev. 1987;3:463-524. 